\title{
A Literature Review on Cross Domain Sentiment Analysis Using Machine learning
}

\author{
Nancy Kansal, Ajay Kumar Garg Engineering College Ghaziabad, India \\ Lipika Goel, Ajay Kumar Garg Engineering College Ghaziabad, India \\ Sonam Gupta, Ajay Kumar Garg Engineering College Ghaziabad, India
}

\begin{abstract}
Sentiment analysis is the field of NLP which analyzes the sentiments of text written by users on online sites in the form of reviews. These reviews may be either in the form of a word, sentence, document, or ratings. These reviews are used as datasets when applied to train a classifier. These datasets are applied in the annotated form with the positive, negative or neutral labels as an input to train the classifier. This trained classifier is used to test other reviews, either in the same or different domains to know like or dislike of the user for the related field. Various researches have been done in single and cross domain sentiment analysis. The new methods proposed are overcoming the previous ones but according to this survey, no methods best suit the proposed work. In this article, the authors review the methods and techniques that are given by various researchers in cross domain sentiment analysis and how those are compared with the pre-existing methods for the related work.
\end{abstract}

\section{KEYWORDS}

Cross-Domain, Domain Adaptation, Machine Learning, Opinion Mining, Sentiment Analysis, Transfer Learning,

\section{INTRODUCTION}

Sentiment analysis is also called opinion mining or emotion AI. Sentiment Analysis is used to know about what people think about things they are consuming from watching a movie to purchasing an AC. On the basis of their thinking either positive or negative, producer or holders of service or product get to know whether the given service/product has the future scope or not. For example, there is a new movie released; various social networking sites are the source of thoughts of people who have seen the movie. On the basis of those reviews, publicity of the movie reaches to the makers of the movie and related trend goes on. Whenever there are elections to be held, election outcomes are predicted on the basis of the analysis of sentiments, opinion, and thoughts those are shared by public on various online portals or social networking sites or news. Sentiment Analysis is the study of attitudes of the holder of service towards the consumed service either in the form of love or hate; like or dislike; positive or negative (polarity). This attitude is analyzed from the text that is presented in the form of reviews in word form, sentence form or document form or in the form of ratings given by holder.

Sentiment Analysis can be done using various machine learning algorithms in which a model/ classifier is trained using reviews that are annotated with the polarity positive or negative. These annotated reviews can be taken from any domain to train the classifier and the trained classifier is tested for the orientation of text or reviews in same or different domain. 
While doing sentiment analysis in single domain, classifier is trained in single domain and is tested for the same domain thus only problem is to annotate the dataset but in cross domain sentiment analysis problem arises that any word/feature having positive meaning in one domain may have negative meaning in other domain; or in one domain, positive sentiments are expressed by some words and in other domain those positive sentiments are expressed using different words(domain specific words in different domains). In the cross domain, the problem of dataset labeling may be a time-consuming and costly process as it is done manually. SentiWordNet can also be a solution as it is an opinion Lexicon derived from WordNet database which is having scores of positive and negative for attributes and hence can tell polarity of the document on the basis of overall polarity of words written in the document, but it is also having a limitation of words. Various methods and techniques have been proposed recently to overcome this problem of labeling of dataset. The aim of the study is to put some relevant studies together in this paper to help the researchers, by comparing the methods proposed in various studies and by also giving a performance comparison of techniques used in studies. This study aims to focus only on the most recent works published during the period 2010 to 2019 .

The remainder of this paper is as follows: Section 2 defines some key Terminologies used to understand the study on cross domain sentiment analysis. Section 3 describes some challenges and issues related to sentiment analysis in the cross domain. Section 4 demonstrates the methodology for this study purpose followed by a brief discussion of methods used in researches those the authors have used in this study. Section 5 demonstrates various datasets used by different researches on cross domain sentiment analysis. Section 6 defines some of the baseline methods used to compare performances of their proposed methods by various scholars. Section 7 gives answers to the questions that are aimed at this survey study. It compares the performance of all the baselines proposed by various researches. Section 8 is about some discussion on methods given to the problem of sentiment analysis in the cross domain and the Section 9 concludes the paper.

\section{KEY TERMINOLOGY}

Here, the authors define some basic terminologies that are used for this review purpose.

\subsection{Domain}

With respect to this research, the domain is such collection where all the entities have similar characteristics like in electronics products, DVD is one domain and AC is a different domain. In social networking sites, Twitter is one domain and Facebook is a different domain.

\subsection{Sentiment Analysis}

Sentiment analysis is the field of natural language processing in which unstructured online public opinions about any product, social media, brand, news, or research and so on presented in the form of reviews, are transformed in structural information that is annotated dataset having the positive, negative, neutral or mixed polarity of sentiments.

Sentiments can be represented in word, sentence or document form and accordingly, analysis is applied on the stated.

\subsubsection{Aspect Based Sentiment Analysis}

When a researcher is interested in the particular feature/aspect along with positive, negative and neutral sentiments of product, to which users are interested in; it is called Aspect Based Sentiment Analysis. For Ex- if someone says "battery of the new phone is short term" then the negative sentiment is shown for the battery of the phone, not for the phone, hence here battery is one aspect of sentence-level sentiment. 


\subsection{Sentiment Analysis Methods}

\subsubsection{Rule/Lexicon-Based Approach}

A lexicon is a collection of words associated with their individual polarity. In this approach, a lexicon is used to detect the polarity of sentiment documents. Like someof the popular lexicons are: AFINN-11, SentiWordNet, and SenticNet. The words of AFINN-11 are manually labeled by Finn Arup Neilsen in 2009-2010, SentiWordNet is augmented form of WordNet having sentiment information of each word, SenticNet provides orientataion associated with nearly 50,000 natural languages concepts.

\subsubsection{Machine Learning-Based Approach}

It has a machine learning classifier that is trained by first input the labeled features then polarity/label of unlabeled features are predicted, either in the same domain or in different domains. The output of this classifier is the polarity of sentiment features of the output domain.

\subsubsection{Cross Domain Sentiment Analysis}

While applying machine learning algorithms for sentiment analysis if model/classifier is trained using the dataset of one domain (called input domain) but is tested with the dataset for a different domain (called output domain), whether that dataset is labeled or unlabeled, then such analysis is called cross domain sentiment analysis. If the dataset is labeled overall sentiment polarity can be found easily but if the dataset is unlabeled, it is tough to predict the overall sentiment of a document. Sentiment analysis is a predominant task in every field that too when it is a smart era of the internet. But it is economically unreliable to do sentiment analysis in every domain, so cross domain sentiment analysis is performed in which classifier is trained in input domain using annotated dataset of that domain and is tested on output domain to annotate the sentiment polarity expressed by the sentiments presented in form of reviews (words, sentences or documents) or ratings as well.

\section{CHALLENGES AND ISSUES IN CROSS DOMAIN SENTIMENT ANALYSIS}

\subsection{Feature Meagerness}

It is the problem when feature those are expressed in the output domain is not found in the input domain. Due to which classifier trained in the input domain is not sufficiently trained for sentiment analysis in the output domain.

\subsection{Polarity Deviation}

When any word in one domain has either positive or negative polarity but in other domains, the same word has opposite polarity then it may cause bad results of the trained classifier as actual sentiments are opposed by the classifier.

\subsection{Lexical Ambiguity}

When a word/feature has different meanings due to different contexts of different domains (as a word has many different meanings based on the context they are being used to) hence classifier trained for input domain may not be accurate for testing in the output domain.

\section{SYSTEMATIC LITERATURE REVIEW}

The authors have performed a systematic literature review to survey current state-of-the-art around cross domain sentiment analysis and based on that work the authors tried to seek the answer to the following two questions. 
Question 1: which method is widely used as a baseline to compare the performance of proposed methods by different authors?

Question 2: which baseline method gives the best comparison results among all the baselines on the basis of their compared performance analysis?

The authors performed this survey on cross domain sentiment analysis, for which the authors followed the following steps:

\subsection{Searching Process}

The authors started this survey by searching for relevant topics for cross domain sentiment analysis. The authors used the Google search engine for this searching process.

\subsection{Sources}

The authors search for digital libraries like IEEE. Google Scholar and ScienceDirect using keywords sentiment analysis cross domain sentiment analysis and cross domain sentiment analysis techniques, as these are keywords to this related review.

\subsection{Study Inclusion Criteria}

The authors have taken research papers mainly during the period 2010 to 2019, related to cross domain sentiment analysis. Table 1 shows a brief discussion of all the studies that the authors have taken for this review.

\subsection{Research Focus}

The authors have performed this research in order to give the answer to two above mentioned questions so that it might aid to researchers to enhance their research in respected field of cross domain sentiment analysis.

The authors have considered all the mentioned papers to give answers to the above questions.

\section{DATASETS TAKEN BY STUDIES}

Datasets collected for most studies (P1, P2, P3, P4, P5, P6, P7, P8, and P9) are in the English language. This one selected study has a Chinese dataset of reviews of restaurants and cameras from the Dianping website. Reviews from the camera are labeled by three experienced persons. The authors have not considered the part of the study related to that dataset for this review process.

Amazon product dataset is mostly used in research studies as this dataset is widely used to perform cross domain sentiment analysis. Table 2 shows a hetero domain dataset of Amazon product reviews.

Other studies are done on different datasets taken from various domains; those are presented in Table 3.

\section{BASELINE METHODS TAKEN BY STUDIES FOR COMPARISON WITH THE PROPOSED}

Various baseline methods are chosen by various research scholars for their studies which the authors have taken for this review process. Those baseline methods are discussed in Table 4. And some of the baseline methods are discussed below. 
Table 1. Summary of selected studies related to Cross Domain Sentiment Analysis

\begin{tabular}{|c|c|c|c|c|c|}
\hline $\begin{array}{l}\text { Publication } \\
\text { (Year) }\end{array}$ & Methodology/Finding & $\begin{array}{l}\text { Proposed } \\
\text { Classifiers }\end{array}$ & Performance/ Result & $\begin{array}{l}\text { Natural } \\
\text { Language } \\
\text { Processing }\end{array}$ & Key \\
\hline $\begin{array}{l}\text { Cross domain } \\
\text { sentiment } \\
\text { classification via } \\
\text { spectral feature } \\
\text { alignment (2010) }\end{array}$ & $\begin{array}{l}\text { SFA algorithm is proposed to reduce } \\
\text { the gap between cross domain } \\
\text { sentiment data. The co-occurrence } \\
\text { matrix is used to gap between } \\
\text { domain-specific words to domain- } \\
\text { independent words. } \\
\text { Features are represented in the form } \\
\text { of a collection of words (Ngrams) } \\
\text { that are labeled with + (positive) } \\
\text { and -1 (negative) polarity based on } \\
\text { all words in Ngrams. A bipartite } \\
\text { graph is constructed to co-align } \\
\text { domain-independent features to } \\
\text { domain-specific features to find } \\
\text { a new feature space. The spectral } \\
\text { clustering algorithm is applied } \\
\text { on feature bipartite graph to align } \\
\text { domain-specific words if they have } \\
\text { more common domain independent } \\
\text { words and vice-versa. These clusters } \\
\text { then represent a new dataset which is } \\
\text { used to train sentiment classifier. }\end{array}$ & $\begin{array}{l}\text { SFA, LSA, NoTransf, } \\
\text { LSA,FALSA }\end{array}$ & $\begin{array}{l}\text { Accuracy of SFA is compared } \\
\text { with NoTransf, LSA, FALSA, } \\
\text { SCL by } 24 \text { tasks on } 2 \text { datasets. the } \\
\text { t-test is done on the comparison } \\
\text { results of two datasets and SFA } \\
\text { out-performs other methods with } \\
0.95 \text { confidence interval. }\end{array}$ & n-gram & $\mathrm{P} 1$ \\
\hline $\begin{array}{l}\text { Cross domain } \\
\text { sentiment } \\
\text { classification using } \\
\text { sentiment sensitive } \\
\text { thesaurus (2013) }\end{array}$ & $\begin{array}{l}\text { Sentiment sensitive thesaurus (SST) } \\
\text { is created to align words having } \\
\text { the same sentiments from different } \\
\text { domains. } \\
\text { SST is used to expand feature vector } \\
\text { (training set) and using this L1 } \\
\text { Logistic regression based binary } \\
\text { classifier is trained which is used to } \\
\text { predict the sentiment of the target } \\
\text { domain. }\end{array}$ & $\begin{array}{l}\text { L1 Regularized } \\
\text { logistic Regression. }\end{array}$ & $\begin{array}{l}\text { Performance varies with varying } \\
\text { thesaurus size. Accuracy } \\
\text { increases with an increase in } \\
\text { thesaurus size. After saturation, } \\
\text { it decreases with an increase } \\
\text { in size. Trained Classifier is } \\
\text { compared with SentiWordNet, } \\
\text { and it performs better grouping } \\
\text { of words that expresses similar } \\
\text { sentiments. }\end{array}$ & $\begin{array}{l}\text { Unigrams } \\
\text { and bigrams } \\
\text { (called lexicon } \\
\text { elements), } \\
\text { ratings (called } \\
\text { sentiment } \\
\text { elements). }\end{array}$ & $\mathrm{P} 2$ \\
\hline $\begin{array}{l}\text { Cross domain } \\
\text { sentiment } \\
\text { classification using } \\
\text { sensitive sentiment } \\
\text { embeddings (2016) }\end{array}$ & $\begin{array}{l}\text { The unsupervised classification } \\
\text { method is used using spectral } \\
\text { embeddings. Domain dependent } \\
\text { features (pivots) are selected to } \\
\text { map in embedded space as close as } \\
\text { possible. Documents having the same } \\
\text { polarity should be embedded close } \\
\text { to each other than a document with } \\
\text { different polarities. }\end{array}$ & $\begin{array}{l}\text { Composite } \\
\text { optimization model } \\
\text { using OO matrices. }\end{array}$ & $\begin{array}{l}\text { Performance is comparable to } \\
\text { SCL and SFA. }\end{array}$ & $\begin{array}{l}\text { pointwise } \\
\text { mutual } \\
\text { information } \\
\text { (PMI) method } \\
\text { is used for } \\
\text { selecting } \\
\text { pivots from the } \\
\text { document. }\end{array}$ & P3 \\
\hline $\begin{array}{l}\text { Cross-domain } \\
\text { sentiment } \\
\text { classification: } \\
\text { An empirical } \\
\text { investigation (2016) }\end{array}$ & $\begin{array}{l}\text { Three datasets are used to compare } \\
\text { performance using three different } \\
\text { classifiers. Datasets are taken as the } \\
\text { first dataset is created using sentiment } \\
140 \text { corpus, second is SemVal dataset } \\
\text { and the third is dataset as three } \\
\text { review domains. The performance } \\
\text { of cross domain classification is } \\
\text { determined by using these datasets by } \\
\text { training the models. } \\
\text { Supervised learning was applied as } \\
\text { classifiers were tested on manually } \\
\text { labeled tweets. } 8 \text { types of Emoticons } \\
\text { were used to label tweets }\end{array}$ & SVM, NB, MNB & $\begin{array}{l}\text { Best performance is gained using } \\
\text { MNB trained by tweets dataset to } \\
\text { determine sentiment in reviews. } \\
\text { The best performance was gained } \\
\text { using SVM with unigrams and } \\
\text { ME with unigrams and bigrams. }\end{array}$ & $\begin{array}{l}\text { Unigram, } \\
\text { bigram, } \\
\text { unigram and } \\
\text { bigrams, } \\
\text { unigrams with } \\
\text { parts-of-speech } \\
\text { (POS) bags. }\end{array}$ & $\mathrm{P} 4$ \\
\hline $\begin{array}{l}\text { Cross-domain } \\
\text { sentiment } \\
\text { classification based } \\
\text { on transfer learning } \\
\text { and adversarial } \\
\text { network (2018) }\end{array}$ & $\begin{array}{l}\text { The shared knowledge Learning and } \\
\text { transfer (SKLT) model is introduced } \\
\text { based on Transfer Learning and } \\
\text { adversarial Networks. Shared and } \\
\text { Private models (bi-GRU) are used } \\
\text { to learn shared sentiment knowledge } \\
\text { and domain-specific knowledge. }\end{array}$ & SKLT, bi-GRU & $\begin{array}{l}\text { Single bi-GRU, SKLT- frozen, } \\
\text { SKLT-adaptation are the contrast } \\
\text { models to compare with. And } \\
\text { SKLT domain adaptation } \\
\text { outperforms. }\end{array}$ & n-grams & P5 \\
\hline $\begin{array}{l}\text { Hierarchical } \\
\text { attention transfer } \\
\text { network (HATN) } \\
\text { for cross product } \\
\text { sentiment } \\
\text { classification (2018) }\end{array}$ & $\begin{array}{l}\text { HATN automatically captures pivot } \\
\text { and non-pivots elements. P-nets and } \\
\text { NP-nets conduct Attention learning to } \\
\text { find pivots and non-pivots elements. } \\
\text { It provides a hierarchical attention } \\
\text { transfer mechanism that automatically } \\
\text { transfers the attention of emotions in } \\
\text { both word and sentence levels across } \\
\text { domains. HATNh is a proposed } \\
\text { model that has hierarchical positional } \\
\text { encoding. }\end{array}$ & $\begin{array}{l}\text { NLTK used for } \\
\text { tokenization, HATN, } \\
\text { HATNh }\end{array}$ & $\begin{array}{l}\text { Comparison is done with the } \\
\text { baseline models like SFA, } \\
\text { DANN, DAmSDA, CNN-aux, } \\
\text { AMN, P-net, NP-net. And it is } \\
\text { found that representation of P-net } \\
\text { and NP-net are complementary. } \\
\text { HATNh improves the } \\
\text { performance of HATN by } 0.41 \% \\
\text { on average. }\end{array}$ & $\begin{array}{l}\text { Document- } \\
\text { based features. }\end{array}$ & P6 \\
\hline
\end{tabular}




\begin{tabular}{|c|c|c|c|c|c|}
\hline $\begin{array}{l}\text { Publication } \\
\text { (Year) }\end{array}$ & Methodology/Finding & $\begin{array}{l}\text { Proposed } \\
\text { Classifiers }\end{array}$ & Performance/ Result & $\begin{array}{l}\text { Natural } \\
\text { Language } \\
\text { Processing }\end{array}$ & Key \\
\hline $\begin{array}{l}\text { Cross Domain } \\
\text { sentiment } \\
\text { classification by } \\
\text { Capsule network } \\
\text { with semantic rules } \\
\text { (2018) }\end{array}$ & $\begin{array}{l}\text { CapsuleDAR Model consist of } \\
\text { two capsules is used. (Called Base } \\
\text { Network and Rule Network). Rule } \\
\text { Network to integrate semantic rule to } \\
\text { capsule network to capture common } \\
\text { knowledge of different domains. } \\
\text { Base Network is having an } \\
\text { embedding layer to convert word } \\
\text { into a low dimensional vector } \\
\text { representation, convolutional layer } \\
\text { to extract n-gram features. Pivot } \\
\text { Based Filter Initialization method is } \\
\text { introduced. } \\
\text { SCL is used to select pivot features. } \\
\text { The K-means method is used to } \\
\text { cluster the features. Incaps, Outcaps, } \\
\text { and classcaps layers are used in Base } \\
\text { Network. } \\
\text { Rulecapes layer is used in Rule } \\
\text { network. CORAL LOSS is used } \\
\text { to minimize the feature difference } \\
\text { between the source and target } \\
\text { domain. }\end{array}$ & $\begin{array}{l}\text { CapsuleDAR } \\
\text { (capsule network in } \\
\text { Domain Adaptation } \\
\text { with semantic Rule) }\end{array}$ & $\begin{array}{l}\text { The model outperforms various } \\
\text { methods like SCL-MI,SS- } \\
\text { FE,DANN, SVM, DACNN, } \\
\text { DAmSDA, AE-SCL-SR, PBLM, } \\
\text { and CapsuleNoDA. It gives a } \\
7.9 \% \text { improvement over its best } \\
\text { competitor (AMN). }\end{array}$ & n-grams & P7 \\
\hline $\begin{array}{l}\text { Adding prior } \\
\text { knowledge In } \\
\text { hierarchical attention } \\
\text { neural network } \\
\text { (HANP) for cross } \\
\text { domain sentiment } \\
\text { classification (2019) }\end{array}$ & $\begin{array}{l}\text { Sentiment Dictionary Layer is used } \\
\text { to identify all sentiment words in the } \\
\text { context of pivots, non-pivots, and } \\
\text { dis-pivots. 3-Layer CNN is used for } \\
\text { contextual preservation from source } \\
\text { Domain to target Domain. HANP } \\
\text { is tested on various datasets for } \\
\text { classification. }\end{array}$ & HANP & $\begin{array}{l}\text { It is compared to HAN, CNN- } \\
\text { aux, AMN, HATNh, HAN+CNN, } \\
\text { HAN+CNN+pivots, } \\
\text { HAN+CNN+pivots+non-pivots } \\
\text { and gives a state-of-the-art } \\
\text { performance with the max. } \\
\text { average accuracy of } 5.78 \% \text { when } \\
\text { compared with the CNN-aux. }\end{array}$ & n-grams & P8 \\
\hline $\begin{array}{l}\text { CCHAN: An end-to- } \\
\text { end model for cross } \\
\text { domain sentiment } \\
\text { classification (2019) }\end{array}$ & $\begin{array}{l}\text { CTN + CTAN = CCHAN. Cloze } \\
\text { Task Network (CTN) is used to } \\
\text { obtain word embeddings and also } \\
\text { matching is done between document } \\
\text { and candidate answer.(to update word } \\
\text { embeddings in the source as well as } \\
\text { target domains). } \\
\text { CTAN is used for sentiment } \\
\text { classification. }\end{array}$ & CCHAN & $\begin{array}{l}\text { Model is compared with HAN, } \\
\text { CNN-aux, AMN, HATN } h \text {, } \\
\text { CHAN, CCHAN-pivots, and it } \\
\text { outperforms all the models. }\end{array}$ & n-gram & P9 \\
\hline $\begin{array}{l}\text { Neural attentive } \\
\text { network for cross- } \\
\text { domain aspect } \\
\text { level sentiment } \\
\text { classification (2019) }\end{array}$ & $\begin{array}{l}\text { It uses a weekly supervised Latent } \\
\text { Dirichlet Allocation Model (Wilda) } \\
\text { to learn Domain-specific Aspect and } \\
\text { sentiment Lexicon representations. } \\
\text { Aspect level sentiment classifier uses } \\
\text { domain classification results and } \\
\text { aspect document representation to } \\
\text { classify aspect level sentiments in the } \\
\text { target domain. } \\
\text { LSTM is used to encode the input } \\
\text { document. NAACL transforms } \\
\text { document embeddings to domain- } \\
\text { specific document embeddings. }\end{array}$ & Bi-directional LSTM & $\begin{array}{l}\text { NAACL is superior to compared } \\
\text { baseline methods in terms of } \\
\text { classification accuracy and F1 } \\
\text { score. And also it is shown that } \\
\text { it can also find the words that are } \\
\text { important to judge the polarity of } \\
\text { the source text. Baseline methods } \\
\text { are SVM, SVM feature, LSTM, } \\
\text { TD-LSTM, JST, SFA, SDA-LSS, } \\
\text { ATAE-LSTM, MemNET, RAM, } \\
\text { IAM. }\end{array}$ & $\begin{array}{l}\text { wsLDA } \\
\text { (weakly } \\
\text { supervised } \\
\text { latent Drichilit } \\
\text { Allocation) } \\
\text { is used to } \\
\text { finddomain- } \\
\text { specific } \\
\text { aspects from } \\
\text { documents. }\end{array}$ & P10 \\
\hline $\begin{array}{l}\text { Cross-domain } \\
\text { co-extraction of } \\
\text { sentiment and topic } \\
\text { lexicons (2012) }\end{array}$ & $\begin{array}{l}\text { A new bootstrapping-based method, } \\
\text { Realtional Adaptive Bootstrapping } \\
\text { (RAP) is proposed for expanding } \\
\text { lexicon to retrain the classifier. } \\
\text { Transfer Adaboost learning } \\
\text { (TrAdaBoost) algorithm (Dai et al., } \\
\text { 2007) is used for learning in RAP. } \\
\text { They have used SVM as a base } \\
\text { classifier in Tr-AdaBoost. }\end{array}$ & $\begin{array}{l}\text { Relational Adaptive } \\
\text { Bootstrapping(RAP), } \\
\text { Tr-AdaBoost, SVM }\end{array}$ & $\begin{array}{l}\text { The relational bootstrapping } \\
\text { method(RAP) performs better } \\
\text { than the TrAdaBoost and the } \\
\text { cross-domain CRF algorithm, and } \\
\text { achieves comparable results with } \\
\text { the semi-supervised method. }\end{array}$ & $\begin{array}{l}\text { POS tagging } \\
\text { is used to } \\
\text { represent } \\
\text { previous, } \\
\text { current and } \\
\text { next words. }\end{array}$ & P11 \\
\hline
\end{tabular}

\subsection{NoTransf}

Transfer learning is the process where a model is trained using a large amount of annotated dataset and this model is used as a baseline to train other data. In (Pan et al., 2010) pan et al. has used the NoTransf classifier that is trained only by training data of the source domain. And is used to test the target domain. 
Table 2. Research studies based on Amazon Product dataset

\begin{tabular}{|l|l|l|l|}
\hline \multicolumn{1}{|c|}{ Dataset } & \multicolumn{1}{|c|}{ Komain } & \multicolumn{1}{c|}{ Author } \\
\hline \multirow{4}{*}{ Amazon product reviews } & \multirow{2}{*}{ DVD, Kitchen, Books, Electronics } & P1: 2010 & Pan et al. \\
\cline { 3 - 4 } & & P2: 2013 & Bollegala et al. \\
\cline { 3 - 4 } & P3: 2016 & Bollegala et al. \\
\cline { 3 - 4 } & P5: 2018 & Xiaoyu Duan et al. \\
\cline { 3 - 4 } & P6: 2018 & Li et al. \\
\cline { 3 - 4 } & P7:2018 & Zhang et al. \\
\cline { 3 - 4 } & P8:2019 & Tu Manshu and Wang Bing \\
\cline { 3 - 4 } & P9:2019 & Tu Manshu and Zhao Xuemin \\
\hline
\end{tabular}

Table 3. Dataset taken by selected research studies

\begin{tabular}{|l|l|l|l|l|}
\hline Key & \multicolumn{1}{|c|}{ Author(year) } & \multicolumn{1}{|c|}{ Dataset } & \multicolumn{1}{c|}{ Domain } & \multicolumn{1}{c|}{ Description } \\
\hline P1 & Pan et al.(2010) & $\begin{array}{l}\text { Yelp and Citysearch } \\
\text { reviews dataset } \\
\text { Amazon product reviews } \\
\text { dataset }\end{array}$ & $\begin{array}{l}\text { Hotel } \\
\text { Videogames } \\
\text { Software } \\
\text { Electronics }\end{array}$ & $\begin{array}{l}\text { 12000 reviews from www.yelp. } \\
\text { com and www.citysearch.com } \\
8000 \text { review from each domain } \\
\text { are taken from www.amazon.com }\end{array}$ \\
\hline P4 & Brian et al.(2016) & $\begin{array}{l}\text { Sentiment140 corpus } \\
\text { dataset } \\
\text { SemEval dataset } \\
\text { Reviews and rating dataset }\end{array}$ & $\begin{array}{l}\text { Twitter tweets } \\
\text { (emotions) } \\
\text { Tweets (emotions) } \\
\text { Hotels } \\
\text { Doctors } \\
\text { Restaurant }\end{array}$ & $\begin{array}{l}\text { From 1.6 million tweets from } \\
\text { www.twitter.com labeled with } \\
\text { emoticons, 10000 were used for } \\
\text { the study. } \\
\text { Manually annotated tweets } \\
\text { Were taken. } \\
\text { 2836 annotated reviews were } \\
\text { taken from. }\end{array}$ \\
\hline P5 & $\begin{array}{l}\text { Xiaoyu Duan et } \\
\text { al.(2018) }\end{array}$ & IMDB reviews dataset & Movies & $\begin{array}{l}\text { Labeled English sentences are } \\
\text { taken. }\end{array}$ \\
\hline P10 & Tang et al.(2019) & $\begin{array}{l}\text { Semeval14, SemEval15 } \\
\text { SemEval16 datasets }\end{array}$ & $\begin{array}{l}\text { Restaurant } \\
\text { Laptop }\end{array}$ & $\begin{array}{l}\text { Five aspect based categories } \\
\text { are used, those are price, } \\
\text { food, service, ambiance and } \\
\text { miscellaneous. } \\
\text { Categorized on the basis of } \\
\text { performance, price, quality, and } \\
\text { appearance. }\end{array}$ \\
\hline
\end{tabular}

\subsection{LSA}

LSA is used to find the features having the same meaning in a review text document. Pan et al. (Pan et al., 2010) used LSA as a baseline method to train the classifier by applying LSA in domain-specific features.

\subsection{FALSA}

Pan et al. (Pan et al., 2010) used FALSA as the base method that works in the same way as LSA except that it applies LSA on the co-occurrence matrix of domain-specific and domain-independent features.

\subsection{No Adapt}

When a classifier is trained, feature expansion is done as preprocessing step to train the classifier but in No adapt baseline method feature extraction is not performed but binary classifier is trained only by using unigram and bigram features from annotated source domain and classifier is tested for the dataset of the target domain. 


\subsection{SFA}

SFA aligns domain-specific features/words from different domains and forms a cluster of those aligned features. In (Bollegala et al., 2016; Li et al., 2018) authors have used SFA as a baseline method on their dataset to compare their proposed method's performances.

\subsection{SVM}

SVM is a discriminant also called hyperplane that separates the annotated features in two different classes in a multidimensional space. A-line/ discriminator is drawn between the two classes. Regularization parameter (c) is used to set the margin of the discriminator such that smaller c value, higher the margin and vice-versa. In (Heredia et al., 2016), Brian et al. have set c to 5.0 for their study and in (Zhang et al., 2018), Zhang et al. has used SVM with RBM kernel.

\subsection{NAÏVE BAYES}

In general, naïve Bayes classifier works on Bayes theorem which works on the relative probability of an outcome ( $\mathrm{p}(\mathrm{x} / \mathrm{E})$ means the probability of $\mathrm{x}$ while event $\mathrm{E}$ occurs). In sentiment analysis, it calculates the probability for features to belong in a particular class/polarity (positive and negative). It is called naïve as it assumes features/input words to be independent of each other. In (Heredia et al., 2016) Brian et al. used NB as a baseline as it can give good performance and shows dependencies of features on local as well as global level.

\subsection{CNN-aux}

It is CNN with two auxiliary tasks to aid sentence embedding. It is used by ( $\mathrm{Li}$ et al., 2018; Manshu et al., 2019) as their baseline method for the same purpose. (Tu Manshu and Wang Bing, 2019) have also used it for sentiment classifiers.

\subsection{AMN}

(Li et al. 2017) proposed AMN that automatically captures pivots using an attention mechanism. It does not need a manual selection of pivots. It consists of two memory networks that were sharing parameter, one for sentiment classification and other for domain Classification. And both networks were jointly trained. Thus AMN was focused to learn pivots only ( $\mathrm{Li}$ et al. 2017). AMN is used by (Tu and Wang, 2019) as the baseline for their study.

\section{$6.10 \mathrm{JST}$}

JST is the extension of latent dirichlet allocation (LDA) that is used for document-level classification as it constructs an additional sentiment layer (Lin et al., 2012). JST is used by (Yang et al., 2019) with the same parameter as taken in its original paper.

\subsection{HAN}

It is used for document classification as it constructs a document vector. For this it first selects important words to form a sentence vector, the sentence vectors are aggregated to form the document vector. (Tu and Wang, 2019) have used HAN in their studies to compare the performance of their proposed models.

\subsection{DANN}

DANN can be applied to almost any feed-forward model by increasing a few standard layers and a gradient reversal layer and the resulting layer is trained (Ganin et al., 2016). 


\section{PERFORMANCE COMPARISON OF BASELINES PROPOSED IN STUDIES}

Based on this comparison study of performance measures of various baselines it is found that all methods taken as baselines give accuracy according to the different parameters and datasets on which those are applied. The performance of methods varies with variation in parameter values and selection of source and target domain combinations. They also depend upon the different feature selection methods applied by different researches. Table 5 . shows the values of performance matrices of different baseline methods that are calculated by researchers in their proposed papers. Performance comparison results pictured in Figure 1 show that although SFA is a widely used method in various studies SKLT gives the best accuracy in all the methods that the authors have studied for sentiment analysis in cross-domain.

\section{DISCUSSION}

Most of the techniques of cross domain sentiment analysis depend upon the similarity of source and target domains. During study an over belief is made upon the similarity of features of source and target domains however as there is meagerness between the features of source and target domains, the techniques give poor results with less accuracy. Furthermore, more accurate results can be found while using labeled datasets to train different models for classification. Labeling dataset manually is costly as well as time-consuming, hence various techniques are being applied by different researches in the last few years. The attention mechanism is introduced that automatically captures pivot features without human intervention. Based on that attentive network is proposed that selects important sentiment from the whole document dynamically and give higher word attention to only domainspecific and domain-independent or pivot whole-part relationships. Based on this review research the authors can classify cross domain sentiment analysis methods or techniques into two classes. The first method is based on the transfer of training data features to testing data features. Example studies of this class are feature-based and thesaurus based researches. The second class is the transfer of the complete document from the target domain to the source domain to work as a training dataset to train the model. An example under this class is active learning-based techniques.

There are different challenges that still need to overcome like polarity deviation and lexical ambiguity. Sentiments in different languages, mixed polarity sentiments, differences in contexts, etc are yet to be faced by the techniques introduced by different researches.

\section{CONCLUSION}

Sentiment analysis has gained a lot of attention from researchers as it is in demand with the increasing online sentiments of users on different topics as it gives the ability to extract insights from the opinions, sentiments, thoughts reviews and online response that is being given by users. Cross domain sentiment analysis is a relevant topic about the same application in which one topic can be used to predict certain decisions about other topics as it provides the facility to train and test sentiments behind heterogeneous topics that can be used to make decisions. Hence, sentiment analysis in the cross domain is widely used as their research topic by many researchers those all to give the solution to this problem by giving models for testing and training based on different methods and techniques to improve the accuracy of results.

The authors have performed this study on a systematic literature review on previous researches related to the same to help the researchers in their respective fields to build a model that gives better performance by knowing the pros and cons of previous related methods proposed. As per this study no technique or method yet proposed gives the perfect solution but later methods proposed are always better than former methods proposed in terms of accuracy. Performance of cross domain sentiment analysis depends on the proper selection of source domain to train the classifier to test the target 
Table 4. Baseline methods used in studies

\begin{tabular}{|c|c|c|c|}
\hline $\begin{array}{c}\text { Baseline comparison } \\
\text { methods }\end{array}$ & Elision & Key & Author (year) \\
\hline No transfer & No-Transf & $\mathrm{P} 1$ & Pan et al. (2010) \\
\hline Latent semantic Analysis & LSA & $\mathrm{P} 1$ & Thomas Hofmann (2001) \\
\hline $\begin{array}{l}\text { Featured latent semantic } \\
\text { Analysis }\end{array}$ & FALSA & $\mathrm{P} 1$ & $\begin{array}{l}\text { Serafin and Di Eugenio } \\
\text { (2004) }\end{array}$ \\
\hline Negative adaptation & No adapt & $\mathrm{P} 2, \mathrm{P} 3$ & \\
\hline $\begin{array}{l}\text { Spectral Feature } \\
\text { Alignment }\end{array}$ & SFA & P3, P6, P10 & Pen et al. (2010) \\
\hline $\begin{array}{l}\text { Structured Correspondence } \\
\text { Learning }\end{array}$ & SCL & $\mathrm{P} 1, \mathrm{P} 3$ & Blitzar et al. (2016) \\
\hline Support Vector Machine & SVM & $\mathrm{P} 4, \mathrm{P} 7, \mathrm{P} 10$ & $\begin{array}{l}\text { Vladimir Vapnik and Hava } \\
\text { Siegelmann (2001) }\end{array}$ \\
\hline Naïve Bayes & NB & P4 & $(1960)$ \\
\hline $\begin{array}{l}\text { Discriminant Adaptive } \\
\text { Nearest Neighbor }\end{array}$ & DANN & P6, P7 & Ganin et al. (2016) \\
\hline $\mathrm{DANN}+\mathrm{mSDA}$ & DAmSD & P6,P7 & Ganin et al. (2016) \\
\hline $\begin{array}{l}\text { Convolutional Neural } \\
\text { Network auxiliary }\end{array}$ & $\mathrm{CNN}$-aux & P6, P8, P9 & Yu and Jiang (2016) \\
\hline $\begin{array}{l}\text { Adversarial Memory } \\
\text { Network }\end{array}$ & $\mathrm{AMN}$ & P6, P8, P9 & Li et al. (2017) \\
\hline SCL Mutual Information & SCL-MI & P7 & Blitzar et al. (2007) \\
\hline $\begin{array}{l}\text { Hierarchical attention } \\
\text { Network }\end{array}$ & HAN & P8, P9 & Yang et al. (2016) \\
\hline $\begin{array}{l}\text { Joint Sentiment Topic/ } \\
\text { Model }\end{array}$ & JST & P10 & Lin et al. (2012) \\
\hline Long Short Term Memory & LSTM & P10 & $\begin{array}{l}\text { S. Hochreiter and J. } \\
\text { Schmidhuber (1997) }\end{array}$ \\
\hline $\begin{array}{l}\text { Stacked Denoising } \\
\text { Autoencoder with Domain } \\
\text { and Sentiment Supervision }\end{array}$ & SDA-DSS & P10 & Liu and Huang (2015) \\
\hline Transfer Ada-Boost & Tr-AdaBoost & P11 & Dai et al. (2007) \\
\hline
\end{tabular}

domain hence proper identification of source domain for a particular domain is most important for feature similarity of domains. The study should be done to select the proper source domain for the adaptation of the target domain.

Techniques should be chosen such as to face all the challenges for sentiment analysis in the cross domain. The most important in which is lexical ambiguity in which word/sentiment's meaning changes with context, hence proper domain selection is required to minimize this ambiguity. 
Table 5. Performance comparisons of baselines proposed in different researches taken for this study

\begin{tabular}{|c|c|c|c|c|c|c|}
\hline Baselines & Key & Accuracy (\%) & F-Score & $\begin{array}{l}\text { AUC- } \\
\text { Score }\end{array}$ & Description & $\begin{array}{c}\text { Average } \\
\text { Accuracies }\end{array}$ \\
\hline SFA(Spectral Feature Alignment) & $\begin{array}{l}\text { P1 } \\
\text { P2 } \\
\text { P3 } \\
\text { P6 } \\
\text { P10 }\end{array}$ & $\begin{array}{l}86.75 \\
77.73 \\
65.47 \\
78.69 \\
78.6\end{array}$ & 78.4 & & $\begin{array}{l}\text { The highest accuracy achieved } \\
\text { when comparing SFA with } \\
\text { different datasets in the cross } \\
\text { domain aspect. } \\
\text { SFA when compared to the } \\
\text { baseline in SST (Bollegala et } \\
\text { al.2013). } \\
\text { With the dimensionality set to } \\
\text { 30, experimented on the Amazon } \\
\text { dataset. } \\
\text { Average acc. On Amazon reviews } \\
\text { dataset. }\end{array}$ & $77.44 \%$ \\
\hline $\begin{array}{l}\text { SCL(Structural Correspondence } \\
\text { Learning) }\end{array}$ & P3 & 66.04 & & & $\begin{array}{l}\text { Average accuracy on target domain } \\
\text { when different source domains } \\
\text { are used. }\end{array}$ & $66.04 \%$ \\
\hline NoAdapt (Negative Adaptation) & P3 & 62.91 & & & $\begin{array}{l}\text { The average accuracy of the } \\
\text { baseline when different source } \\
\text { domains are used to compare } \\
\text { the performance of the proposed } \\
\text { methods. }\end{array}$ & $62.91 \%$ \\
\hline NB(Naïve Bayes) & $\mathrm{P} 4$ & - & & 0.764 & $\begin{array}{l}\text { When using the sentiment } 140 \\
\text { Amazon reviews dataset. }\end{array}$ & \\
\hline SVM(Support Vector Machine) & $\begin{array}{l}\text { P4 } \\
\text { P7 } \\
\text { P10 }\end{array}$ & $\begin{array}{l}- \\
80.2 \\
72.7\end{array}$ & 70.9 & 0.780 & $\begin{array}{l}\text { Using sentiment } 140 \text { corpus } \\
\text { Amazon reviews dataset. } \\
\text { Zhang et al. selected } \\
\text { hyperparameter c between } 10^{\wedge}-5 \\
\text { to } 1 \text {. }\end{array}$ & $76.45 \%$ \\
\hline SKLT & P5 & 87.98 & & & $\begin{array}{l}\text { Average accuracy to compare } \\
\text { performance in adversarial } \\
\text { networks.(Duan et al.2019) }\end{array}$ & $87.98 \%$ \\
\hline $\begin{array}{l}\text { DANN(Discriminant Adaptive } \\
\text { Nearest Neighbor) }\end{array}$ & $\begin{array}{l}\text { P6 } \\
\text { P7 }\end{array}$ & $\begin{array}{l}79.00 \\
74.8\end{array}$ & & & $\begin{array}{l}\text { Average classification accuracy on } \\
\text { Amazon review datasets. Encoded } \\
\text { in } 5000 \text { dimension feature vector. } \\
\text { Average accuracy When tested } \\
\text { on } 12 \text { different sets of domains } \\
\text { using the adaptation parameter } \\
\text { between } 0.001 \text { and } 1 \text { with learning } \\
\text { rate } 0.001 \text {. }\end{array}$ & $76.9 \%$ \\
\hline $\mathrm{DANN}+\mathrm{mSDA}$ & $\begin{array}{l}\text { P6 } \\
\text { P7 }\end{array}$ & $\begin{array}{l}82.36 \\
76.2\end{array}$ & & & $\begin{array}{l}\text { Average acc. Using Amazon } \\
\text { review dataset with } 5 \text { output layers } \\
\text { and a vector of } 30000 \text { dimensions. } \\
\text { Average accuracy on } 12 \text { different } \\
\text { domain sets with every instance } \\
\text { encoded in a vector of } 3000 \\
\text { dimensions. }\end{array}$ & $79.28 \%$ \\
\hline CNN-aux & $\begin{array}{l}\text { P6 } \\
\text { P8 } \\
\text { P9 }\end{array}$ & $\begin{array}{l}81.98 \\
81.98 \\
81.98\end{array}$ & & & $\begin{array}{l}\text { Average accuracy for Amazon } \\
\text { reviews dataset to induce } \\
\text { sentiment embeddings using two } \\
\text { auxiliary tasks. } \\
\text { Average accuracy when using } 20 \\
\text { sets of different source and target } \\
\text { domains. } \\
\text { Average accuracy for } 20 \text { transfer } \\
\text { pairs of Amazon review dataset. }\end{array}$ & $81.98 \%$ \\
\hline $\begin{array}{l}\text { AMN(Adversarial Neural } \\
\text { Network) }\end{array}$ & $\begin{array}{l}\text { P6 } \\
\text { P8 } \\
\text { P9 }\end{array}$ & $\begin{array}{l}82.79 \\
82.79 \\
82.79\end{array}$ & & & $\begin{array}{l}\text { Average classification accuracy for } \\
\text { Amazon review dataset by learning } \\
\text { domain shared representations. } \\
\text { Average acc. When taken } 20 \\
\text { different domain pairs for study. } \\
\text { Average acc. For } 20 \text { transfer pairs } \\
\text { on Amazon review dataset. }\end{array}$ & $82.79 \%$ \\
\hline $\begin{array}{l}\text { HAN(Hierarchical Attention } \\
\text { Network) }\end{array}$ & $\begin{array}{l}\text { P8 } \\
\text { P9 }\end{array}$ & $\begin{array}{l}81.07 \\
81.07\end{array}$ & & & $\begin{array}{l}\text { Average acc. When taken } 20 \\
\text { different domain pairs for study. } \\
\text { Average acc. For } 20 \text { transfer pairs } \\
\text { on Amazon review dataset. }\end{array}$ & $81.07 \%$ \\
\hline JST & P10 & 79.3 & & & $\begin{array}{l}\text { The performance of this baseline } \\
\text { is used for comparing performance } \\
\text { of NAACL. Performance(given } \\
\text { average accuracy) is measured by } \\
\text { varying percentage of labeled data } \\
\text { in target domain. Labeled data } \\
\text { from source domain and labeled/ } \\
\text { unlabeled data from target domain } \\
\text { are used as training set. }\end{array}$ & $79.3 \%$ \\
\hline
\end{tabular}


Table 5. Continued

\begin{tabular}{|c|c|c|c|c|c|c|}
\hline Baselines & Key & Accuracy (\%) & F-Score & $\begin{array}{l}\text { AUC- } \\
\text { Score }\end{array}$ & Description & $\begin{array}{c}\text { Average } \\
\text { Accuracies }\end{array}$ \\
\hline LSTM & P10 & 78.6 & & & $\begin{array}{l}\text { Average accuracy while taking } \\
\text { the SemEval-14 S-res./Dianping } \\
\text { D-res. as the } \\
\text { source domains and use } \\
\text { SemEval-14 S-laptop/Dianping } \\
\text { D-camera } \\
\text { as the target domains,by varying } \\
\text { percentage of labeled data in target } \\
\text { domain. }\end{array}$ & $78.6 \%$ \\
\hline SDA-DSS & P10 & 81.5 & & & $\begin{array}{l}\text { Average accuracy while taking } \\
\text { the SemEval-14 S-res./Dianping } \\
\text { D-res. as the } \\
\text { source domains and use } \\
\text { SemEval-14 S-laptop/Dianping } \\
\text { D-camera } \\
\text { as the target domains,by varying } \\
\text { percentage of labeled data in target } \\
\text { domain. }\end{array}$ & $81.5 \%$ \\
\hline Tr-AdaBoost & P11 & & 0.51 & & $\begin{array}{l}\text { Average F-Score while taking } \\
\text { two tasks: Sentiment Lexicon } \\
\text { extraction and Topic Lexicon } \\
\text { extraction, on product and movie } \\
\text { review datasets. }\end{array}$ & \\
\hline
\end{tabular}

Figure 1. Performance comparison of baselines using the line graph

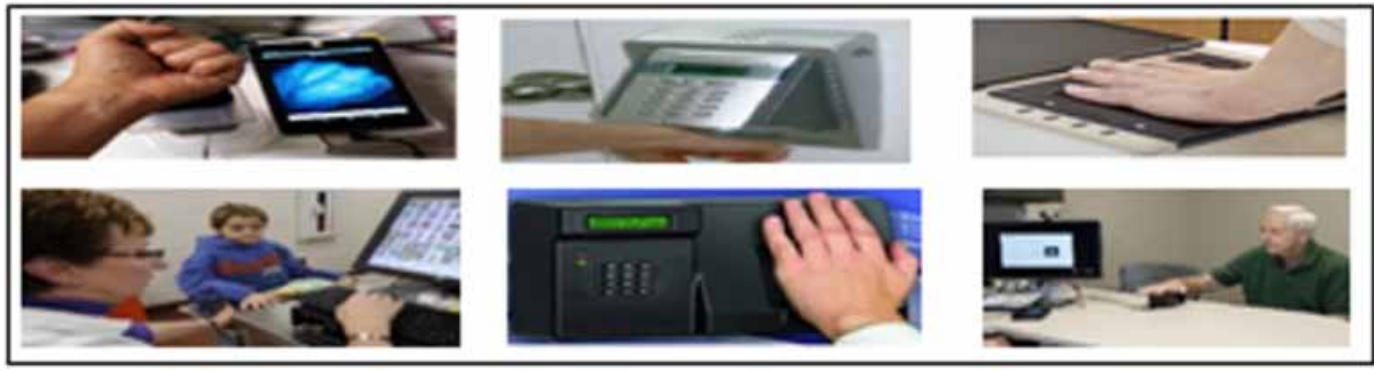




\section{REFERENCES}

Ben-Hur, A., Horn, D., Siegelmann, H. T., \& Vapnik, V. (2001). Support vector clustering. Journal of Machine Learning Research, 2(December), 125-137.

Bollegala, D., Mu, T., \& Goulermas, J. Y. (2016). Cross-Domain Sentiment Classification Using Sentiment Sensitive Embeddings. IEEE Transactions on Knowledge and Data Engineering, 28(2), 398-410. doi:10.1109/ TKDE.2015.2475761

Bollegala, D., Weir, D., \& Carroll, J. (2013). Cross-Domain Sentiment Classification Using a Sentiment Sensitive Thesaurus. IEEE Transactions on Knowledge and Data Engineering, 25(8), 1719-1731. doi:10.1109/ TKDE.2012.103

Dai, W., Yang, Q., Xue, G., \& Yu, Y. (2007). Boosting for transfer learning. In Proceedings of the 24th International Conference on Machine Learning (pp. 193-200). ACM.

Duan, X., Zhou, Y., Jing, C., Zhang, L., \& Chen, R. (2018). Cross-domain Sentiment Classification Based on Transfer Learning and Adversarial Network. In Proceedings of the 2018 IEEE 4th International Conference on Computer and Communications (ICCC) (pp. 2302-2306). IEEE Press. 10.1109/CompComm.2018.8780771

Ganin, Y., Hana, A. H. L., Laviolette, F., \& Lempitsky, V. (2016). Domain-Adversarial Training of Neural Networks. Journal of Machine Learning Research, 17, 1-35.

Heredia, B., Khoshgoftaar, T. M., Prusa, J., \& Crawford, M. (2016). Cross-Domain Sentiment Analysis: An Empirical Investigation. In Proceedings of the 2016 IEEE 17th International Conference on Information Reuse and Integration (IRI) (pp. 160-165). IEEE Press. 10.1109/IRI.2016.28

Hochreiter, S., \& Schmidhuber, J. (1997). Long Short Term Memory. Journal Neural Computation, 9(8), 1735-1780. doi:10.1162/neco.1997.9.8.1735

Hofmann, T. (2001). Unsupervised Learning by Probabilistic Latent Semantic Analysis. Machine Learning, 42(1-2), 177.

Li, F., Pan, S. J., Jin, O., Yang, Q., \& Zhu, X. (2012, July). Cross-domain co-extraction of sentiment and topic lexicons. In Proceedings of the 50th Annual Meeting of the Association for Computational Linguistics (Vol. 1, pp. 410-419). Association for Computational Linguistics.

Li, Z., Wei, Y., Zhang, Y., \& Yang, Q. (2018, April). Hierarchical attention transfer network for cross-domain sentiment classification. In Proceedings of the Thirty-Second AAAI Conference on Artificial Intelligence. AAAI Press.

Li, Z., Zhang, Y., Wei, Y., Wu, Y., \& Yang, Q. (2017, August). End-to-End Adversarial Memory Network for Cross-domain Sentiment Classification. In IJCAI (pp. 2237-2243). Academic Press.

Lin, C., He, Y., Everson, R., \& Ruger, S. (2012, June). Weakly Supervised Joint Sentiment-Topic Detection from Text. IEEE Transactions on Knowledge and Data Engineering, 24(6), 1134-1145. doi:10.1109/TKDE.2011.48

Manshu, T., \& Bing, W. (2019). Adding Prior Knowledge in Hierarchical Attention Neural Network for Cross Domain Sentiment Classification. IEEE Access : Practical Innovations, Open Solutions, 7, 32578-32588. doi:10.1109/ACCESS.2019.2901929

Manshu, T., \& Xuemin, Z. (2019). CCHAN: An End to End Model for Cross Domain Sentiment Classification. IEEE Access : Practical Innovations, Open Solutions, 7, 50232-50239. doi:10.1109/ACCESS.2019.2910300

Pan, S. J., Ni, X., Sun, J. T., Yang, Q., \& Chen, Z. (2010, April). Cross-domain sentiment classification via spectral feature alignment. In Proceedings of the 19th international conference on World wide web (pp. 751760). Academic Press.

Serafin, R., \& Eugenio, B. D. (2004). FLSA: Extending Latent Semantic Analysis with Features for Dialogue Act Classification. ACL. 10.3115/1218955.1219043

Yang, M., Yin, W., Qu, Q., Tu, W., Shen, Y., \& Chen, X. (2019). Neural attentive network for cross-domain aspect-level sentiment classification. IEEE Transactions on Affective Computing. 
Zhang, B., Xu, X., Yang, M., Chen, X., \& Ye, Y. (2018). Cross-Domain Sentiment Classification by Capsule Network with Semantic Rules. IEEE Access : Practical Innovations, Open Solutions, 6, 58284-58294. doi:10.1109/ACCESS.2018.2874623

Nancy Kansal is a young and dynamic academician in the field of Computer Science. She received her B.Tech (Hons.) degree in Computer Science and Engineering from Radha Govind Group of Institutions in 2016. She completed her schooling with First class in Higher Secondary and in Secondary. Currently, she is pursuing her M. Tech in Computer Science and Engineering from Ajay Kumar Garg Engineering College (Ghaziabad) India which is affiliated to Dr. A. P. J. Abdul Kalam Technical University Uttar Pradesh, Lucknow, India. Her research interests include Machine Learning. She has a deep interest in research and innovations and their applications. She is a quick learner and dedicated to the task given to her. She is always passionate to learn new things. Her strength is her positive attitude and deep faith in God.

Lipika Goel has a B.Tech., an M.Tech. and persuing a PhD. She has over 10 years of experience in teaching. Currently, she is working as an Assistant Professor (CSE) in Ajay Kumar Garg Engineering College, Ghaziabad. She has published over 10 papers in various national/international journals. She is reviewer of many international journals/conferences. Her research interest include machine learning and data analytics.

Sonam Gupta has B.E., an Mtech, and a Ph.D. She has over 10 years of experience in teaching. Currently, she is working as an Associate Professor (CSE) in Ajay Kumar Garg Engineering College, Ghaziabad. She has published over 20 papers in various national/international journals. She is a reviewer of many international journals/ conferences. Her research interest includes software evolution, machine learning, and data analytics. 